\title{
Maternal body mass index, neonatal lung function and respiratory symptoms in childhood
}

\author{
Jacobien B. Eising ${ }^{1}$, Cuno S.P.M. Uiterwaal ${ }^{2}$ and Cornelis K. van der Ent ${ }^{1}$
}

Affiliations: ${ }^{1}$ Dept of Paediatric Pulmonology, Wilhelmina Children's Hospital, University Medical Center, Utrecht, The Netherlands. ${ }^{2}$ Julius Center for Health Sciences and Primary Care, University Medical Center, Utrecht, The Netherlands.

Correspondence: Jacobien B. Eising, Paediatric Pulmonology Department, Wilhelmina Children's Hospital, Room KH.01.419.0, Lundlaan 6, PO Box 85090, 3508 AB Utrecht, The Netherlands.

E-mail: j.eisingdumcutrecht.nl

ABSTRACT Recent studies have shown that maternal obesity is associated with increased risk of wheezing in the offspring. We assessed whether impaired neonatal lung function could explain this association.

We measured neonatal lung function in 2606 children of our prospective birth cohort. Information about daily symptoms of wheezing was obtained using questionnaires. Consultations and prescriptions for wheezing illnesses were derived from general practitioner patient files.

Higher maternal body mass index (BMI) was associated with increased risk of wheezing in the first year of life and more consultations and prescriptions for wheezing illnesses until the age of 5 years. Lung function could partially explain the association with wheezing in the first year of life. Adding respiratory resistance to the model decreased the incidence rate ratio from 1.023 (95\% CI 1.008-1.039) to 1.015 (95\% CI 0.998-1.032). Anthropometrics of the 5-year-olds largely explained the association with consultations. Intermediates or confounders could not explain the association with prescriptions.

There is an association between higher maternal BMI and increased risk of wheezing illnesses. In the first year of life, it is largely explained by an impaired lung function in early life, especially in children of nonatopic mothers. At the age of 5 years, infant lung function is of minor influence in this association.

@ERSpublications

Higher maternal BMI is associated with higher risk of wheezing illnesses partly due to impaired neonatal lung function http://ow.ly/OrlHB

\footnotetext{
This article has supplementary material available from erj.ersjournals.com

Received: April 282014 | Accepted after revision: June 022015 | First published online: Aug 202015

Support statement: The Wheezing Illnesses Study Leidsche Rijn received grant 2100.0095 from the Netherlands Organisation for Health Research and Development (ZonMw). An unrestricted grant was received from GlaxoSmithKline. Funding information for this article has been deposited with FundRef.
}

Conflict of interest: Disclosures can be found alongside the online version of this article at erj.ersjournals.com

Copyright OERS 2015 


\section{Introduction}

Maternal pre-pregnancy obesity is associated with adverse health outcomes for the offspring. Children of mothers with a higher body mass index (BMI) have an increased risk of obesity, high blood pressure and diabetes mellitus type 2 in childhood, and even long-term consequences on cardiovascular health are described [1-4]. With an increasing prevalence of both obesity and asthma, there is growing interest in the association between maternal weight and respiratory symptoms [5-8]. Several studies have shown that increased maternal weight and BMI are associated with an increased risk of wheezing in offspring in the first years of life, independent of several confounders $[5,6,8]$. The underlying mechanism of these associations is unknown. The association could not be explained by the child's growth, infectious or atopic mechanisms and was not mediated by obesity-related pregnancy complications $[5,9]$.

Previous studies suggested that leptin, a hormone that is primarily produced by adipocytes could play a role in the mechanism underlying these associations $[7,9]$. Leptin receptors have been identified in the human bronchial and alveolar epithelial cells, bronchial smooth muscle cells and bronchial submucosa. In a recent study we have shown an association between higher leptin plasma concentration and lower lung function (forced expiratory volume in $1 \mathrm{~s}(\mathrm{FEV})$ ) in healthy children, which suggests a functional role for this hormone in the respiratory system [10]. Children of mothers with increased fat mass are probably exposed to higher circulating levels of leptin, which may have consequences for development of the respiratory system. Children with an impaired lung function in early life are more likely to develop wheezing illnesses [11].

We examined in our population-based prospective birth cohort study the association between maternal BMI and respiratory symptoms and the use of medication for respiratory symptoms in the offspring, and assessed whether lung function in early life could be an explanatory factor in these associations.

\section{Methods}

\section{Setting and participants}

The present study is part of the Wheezing Illnesses Study Leidsche Rijn (WHISTLER), a population-based birth cohort study on the determinants and prediction of wheezing illnesses. The study design and rationale of WHISTLER have been described in detail elsewhere [12]. Briefly, healthy infants born in a newly developed residential area in the Netherlands (Leidsche Rijn) were invited to participate. Exclusion criteria were gestational age $<36$ weeks, major congenital abnormalities and neonatal respiratory disease.

The paediatric medical ethics committee of the University Medical Center Utrecht (Utrecht, the Netherlands) approved the study. Written informed consent was obtained from the parents.

\section{Visit in infancy}

Parents visited our outpatient clinic with their offspring at the age of 3-8 weeks. Information on pre- and postnatal risk factors was obtained by questionnaire. The weight of the child was measured using a standard electronic scale and body length using an (infant) stadiometer. Information on the parents was obtained from the Utrecht Health Project (UHP), a large health-monitoring study of all the inhabitants of Leidsche Rijn [13]. 969 fathers and 1128 mothers of the 2686 children participated in this study. The parents who did not participate in the UHP were asked to fill in a questionnaire about their health, including self-reported weight and height.

Lung function was measured using the single occlusion technique during natural sleep. It measures the resistance $(R \mathrm{rs})$, compliance $(C \mathrm{rs})$ and time constant $(\tau \mathrm{rs})$ of the total respiratory system in the absence of respiratory muscle activity [14]. Details of performance of these measurements have been described previously $[14,15]$. Measurements were performed according to the criteria of the American Thoracic Society (ATS)/European Respiratory Society (ERS) task force on infant lung function [16, 17]. At least three technically acceptable occlusions were used to calculate mean $C r s, R$ rs and $\tau$ rs. Inter-rater reliability studies were performed as part of WHISTLER and showed satisfactory quality of lung function measurements [15].

\section{Follow-up data in the first year}

1-year follow-up for symptoms of wheeze and cough after infant lung function measurement was achieved by a daily questionnaire completed by the parents in a logbook. Parents were carefully instructed at the time of lung function measurements by one of the investigators on how to recognise the various respiratory sounds. Daily complaints of wheeze and cough were measured using the questions: "Did your child wheeze today (whistling sound from the chest, not from the upper airways/throat)?" Further questions were asked about anthropometrics and environmental factors, such as feeding pattern, passive smoking and daycare attendance. New questionnaires and reinforcements to complete them were sent on a monthly basis to the parents. If parents still failed to return the questionnaire, they were contacted by telephone. To quantify respiratory symptoms, number of days with wheeze in the first year of life was counted. 
Visit at the age of 5 years

At the age of 5 years the children were invited for a follow-up visit. Prior to this visit, parents were asked to complete a questionnaire about current health and risk factors of their child. During the visit, weight and height were measured and spirometry was performed according to the latest ATS/ERS statement for lung function measurements in preschool children [18]. At least two reproducible flow-volume curves were obtained. The greatest FEV1 was selected. Between November 2007 and November 2011 lung function was measured using a heated Lilly head pneumotachometer system (Viasys Healthcare, Hochberg, Germany). From November 2011 onward, all children were measured using the ZAN spirometer (ZAN 100; nSpire Health Inc., Oberthulba, Germany). A previous study showed no significant differences in FEV1 and forced vital capacity (FVC) between devices of these manufacturers [19].

\section{Medication use and diagnosis of respiratory symptoms}

Data on primary care visits and prescriptions from birth until the age of 5 years were obtained from the general practitioners' (GP) electronic patient files (Medicom; PharmaPartners, Oosterhout, the Netherlands), using standardised codes. The GPs used the International Classification of Primary Care (ICPC) codes for every consultation. The ICPC codes dyspnoea (R02), wheezing (R03) and asthma (R96) were used for a diagnosis of wheezing illness. Medication was classified according to the Anatomical Therapeutic Chemical classification. To quantify consultations and prescriptions for respiratory illnesses, number of diagnoses of lower respiratory symptoms and wheezing illnesses and number of prescriptions for short-acting $\beta_{2}$-agonists and inhaled corticosteroids until the age of 5 years were counted.

\section{Statistical analysis}

Central estimators and variance measures to describe general characteristics were calculated. All variables were checked for normality of distribution. Differences in characteristics of pregnancy and of the offspring across quartiles of increasing maternal BMI were tested using ANOVA for continuous variables and Chi-squared for categorical variables.

The number of days with wheezing between the second and twelfth month of age was used as a count-type outcome, best fitting a negative binomial distribution, as there were many children with no days of wheezing symptoms. We constructed a univariable model to investigate the relation between maternal BMI and number of days with wheeze. Subsequently, multivariable negative binomial regression models were constructed to investigate whether maternal BMI was independently related to number of days with wheeze or cough. We adjusted for intermediates or potential confounders. Selection of confounders was based on previous literature. We adjusted for daycare, breastfeeding, older siblings, smoke exposure during pregnancy, pet keeping, socioeconomic status, ethnicity and maternal age.

Birth weight and infant lung function were added to this model with potential confounders to examine whether these factors could explain an association between maternal BMI and wheezing illnesses.

Selective loss to follow-up could bias our results, as returning questionnaires every month requires effort by the parents. To prevent bias associated with missing data, missing values of determinants (including confounders and intermediates) and the outcome were multiple-imputed based on the correlation of the missing variables with other characteristics. 25 imputed datasets were created and analysed separately, after which results were pooled. We performed the same negative binomial analysis on the imputed data.

The number of consultations and prescriptions up to the age of 5 years were also used as count data, best fitting a negative binomial distribution. We performed similar multivariable regression models with these data, but instead of birthweight, we adjusted for actual weight and height at the 5-years visit.

Finally, we assessed whether maternal atopy could be an effect modifier of this association. Therefore, we stratified the data by maternal atopy and performed the same analyses as described earlier. To test if the modification was significant we added the product of maternal atopy and BMI as an interaction term to the multivariable regression models.

Results are presented as incidence rate ratio (IRR) with 95\% confidence intervals and p-values. Intervals not including 1 and $\mathrm{p}$-values $<0.05$ were considered statistically significant. Data analyses were performed in SPSS (version 20.0; IBM, Armonk, NY, USA).

\section{Results}

An overview of the recruitment and inclusion of participants of the WHISTLER project is shown in figure 1. 2686 children participated in WHISTLER. Monthly questionnaires on $75.0 \%$ of all eligible infants were completed up to the age of 1 year. Data on consultations and prescriptions were available for $76.1 \%$ of all children who reached the age of 5 years. To detect possible confounders, table 1 presents the baseline characteristics of these children per maternal BMI quartile. The mean maternal BMIs in the first, second, 


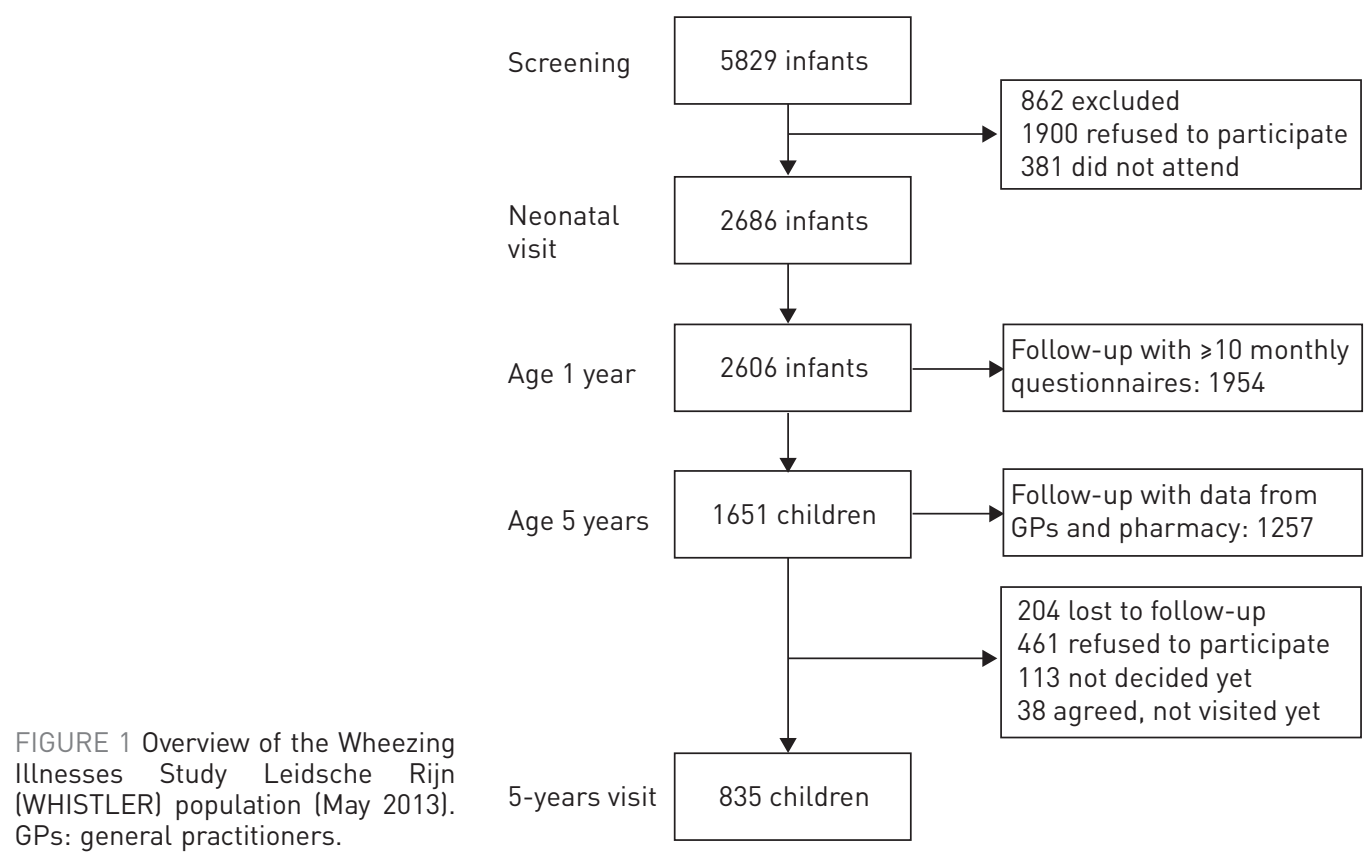

third and fourth quartile were 20.5, 23.0, 25.2 and $30.0 \mathrm{~kg} \cdot \mathrm{m}^{-2}$, respectively. Mothers with a higher BMI were more exposed to smoke during pregnancy $(p<0.01)$. Infants of mothers with a higher maternal BMI had a higher birth length $(\mathrm{p}<0.01)$. Birth weight and weight at the visit at the age of 5 years were also higher in children of mothers with a higher maternal BMI (both $\mathrm{p}<0.01$ ). Children of mothers in the highest quartile were less exposed to breastfeeding $(\mathrm{p}<0.01)$. There were no differences in postnatal growth, lung function or other environmental factors. In online supplementary table S1 characteristics of the participants at baseline and at the age of 5 years are compared. No large differences in these groups were shown.

Table 2 shows the results of the negative binomial regression analysis with number of wheezing days in the first year of life as the dependent variable. In the complete case analyses, every $1 \mathrm{~kg} \cdot \mathrm{m}^{-2}$ increase in maternal BMI was associated with 2.3\% more wheezing days (95\% CI 1.008-1.039\%) after adjustment for potential confounders. Birthweight did not explain the association. Adding Rrs decreased the IRR to 1.015 (95\% CI 0.998-1.032). The univariable model and multivariable models of the imputed data showed no clear associations. Adjustment for birth length or gestational age did not affect the associations (online supplementary table S2). Table 3 shows the results of the imputed data stratified by maternal atopy, which showed that associations between maternal BMI and number of wheezing days are present in children of nonatopic mothers. Every $1 \mathrm{~kg} \cdot \mathrm{m}^{-2}$ increase in maternal BMI in these nonatopic mothers was associated with $2.0 \%$ (95\% CI 1.000-1.042\%) more wheezing in their children. Adding resistance to the model decreased the IRR to 1.010 (95\% CI 0.988-1.033).

Table 4 shows the association between maternal BMI and consultations and prescriptions for respiratory symptoms up to the age of 5 years. With each $1 \mathrm{~kg} \cdot \mathrm{m}^{-2}$ in maternal BMI, 3.3\% (95\% CI $1.001-1.065 \%$ ) more consultations for wheezing illnesses were present after adjustment for potential confounders. Current weight and height of the child at the age of 5 years explained the association with wheezing illnesses (IRR 1.002 , 95\% CI 0.962-1.044). Lung function in early life could partially explain the association with wheezing illnesses (IRR 1.022, 95\% CI 0.986-1.059). Higher maternal BMI was also associated with more prescriptions of short-acting $\beta_{2}$-agonists and inhaled corticosteroids in the offspring. These associations could not be explained by potential confounders, current status of weight and height or lung function in early life.

Table 5 presents the stratified analysis of associations between maternal BMI and consultations and prescriptions for respiratory symptoms up to the age of 5 years. Maternal BMI was only associated with wheezing illnesses among mothers with a history of atopy, but only in the univariable model. The associations with prescriptions of inhaled corticosteroids or short-acting $\beta_{2}$-agonists in children of an atopic mother were stronger compared to the children of nonatopic mothers and could not be explained by current weight and height or infant lung function (all $\mathrm{p}<0.01$ ), while in nonatopic mothers current weight and height or infant lung function did explain the association. 
TABLE 1 Pregnancy and child characteristics by quartiles of maternal body mass index (BMI)

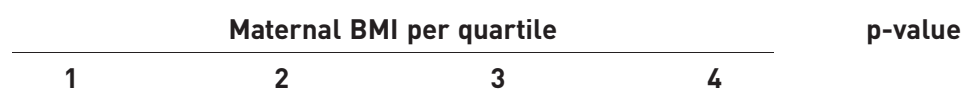

\section{Maternal and pregnancy characteristics}

Maternal age at birth years

Gestational age days

Maternal atopy

Maternal European ethnicity

Smoke exposure during pregnancy

Female

Birthweight $\mathrm{g}$

Birth length $\mathrm{cm}$

Weight growth during the first 3 months of life g.day ${ }^{-1}$

Length growth during the first 3 months of life $\mathrm{cm} \cdot \mathrm{day}^{-1}$

Crs $\mathrm{mL} \cdot \mathrm{kPa}^{-1}$

$\operatorname{Rrs~kPa} \cdot \mathrm{L}^{-1} \cdot \mathrm{s}^{-1}$

Older siblings

Daycare during the first year of life

None

0-3 months

4-6 months

$>6$ months

Duration of breastfeeding

None

0-3 months

4-6 months

$>6$ months

Pet keeping

Child characteristics at 5 -year visit $(n=680$ )

Weight

Height

FEV1 L
Maternal BMI $\mathrm{kg} \cdot \mathrm{m}^{-2}$

Higher education ${ }^{\#}$

Child characteristics at neonatal visit $(n=2257)$

\begin{tabular}{|c|c|c|c|c|}
\hline $20.5 \pm 1.1$ & $23.0 \pm 0.6$ & $25.2 \pm 0.8$ & $30.0 \pm 3.2$ & $<0.01$ \\
\hline $32.7 \pm 3.7$ & $32.7 \pm 3.6$ & $32.8 \pm 3.6$ & $33.0 \pm 3.8$ & 0.52 \\
\hline $278.3 \pm 9.6$ & $279.2 \pm 9.6$ & $278.2 \pm 10.0$ & $278.9 \pm 9.8$ & 0.29 \\
\hline 384 (69.1) & 400 (74.5) & 390 (71.2) & 319 (59.7) & 0.52 \\
\hline 210 (37.5) & 215 (38.9) & 204 (36.9) & 235 (42.5) & 0.22 \\
\hline 466 (83.4) & 462 (83.5) & 486 (87.4) & 474 (86.0) & 0.17 \\
\hline $57(10.1)$ & 63 (11.2) & 69 (12.3) & $96(17.1)$ & $<0.01$ \\
\hline 303 (53.6) & 292 (51.9) & 280 (49.6) & 276 (48.9) & 0.37 \\
\hline $3451 \pm 475$ & $3528 \pm 485$ & $3512 \pm 499$ & $3634 \pm 542$ & $<0.01$ \\
\hline $50.6 \pm 2.2$ & $50.9 \pm 2.2$ & $50.8 \pm 2.4$ & $51.1 \pm 2.3$ & $<0.01$ \\
\hline $28.8 \pm 5.2$ & $29.2 \pm 5.6$ & $28.8 \pm 5.6$ & $28.9 \pm 5.2$ & 0.71 \\
\hline $0.12 \pm 0.01$ & $0.12 \pm 0.01$ & $0.12 \pm 0.01$ & $0.12 \pm 0.01$ & 0.30 \\
\hline $46.5 \pm 11.0$ & $46.9 \pm 11.0$ & $46.0 \pm 11.3$ & $47.0 \pm 12.0$ & 0.51 \\
\hline $6.2 \pm 2.1$ & $6.6 \pm 2.1$ & $6.7 \pm 1.9$ & $6.7 \pm 2.0$ & 0.69 \\
\hline 312 (55.3) & 302 (53.7) & 319 (56.9) & 296 (52.7) & 0.52 \\
\hline & & & & 0.12 \\
\hline 153 (29.2) & 132 (26.2) & 120 (23.3) & 151 (30.1) & \\
\hline 29 (5.5) & $24(4.8)$ & $23(4.5)$ & 21 (4.2) & \\
\hline 59 (11.3) & 50 (9.9) & 49 (9.5) & $37(7.4)$ & \\
\hline \multirow[t]{2}{*}{283 (54.0) } & 297 (59.0) & 323 (62.7) & 293 (58.4) & \\
\hline & & & & $<0.01$ \\
\hline 102 (18.4) & 96 (17.4) & 108 (19.6) & 163 (29.5) & \\
\hline 237 (42.9) & $258(46.8)$ & $258(46.7)$ & 244 (44.2) & \\
\hline 119 (21.5) & 140 (25.4) & 110 (19.9) & 91 (16.5) & \\
\hline 95 (17.2) & 57 (10.3) & 76 (13.8) & 54 (9.8) & \\
\hline 196 (34.7) & $206(36.6)$ & 217 (38.4) & 225 (39.9) & 0.30 \\
\hline $19.4 \pm 2.3$ & $20.1 \pm 2.5$ & $20.3 \pm 2.8$ & $20.7 \pm 3.1$ & $<0.01$ \\
\hline $114.8 \pm 5.0$ & $115.6 \pm 4.6$ & $115.5 \pm 5.2$ & $115.1 \pm 4.6$ & 0.48 \\
\hline $1.24 \pm 0.20$ & $1.25 \pm 0.23$ & $1.31 \pm 0.76$ & $1.29 \pm 0.26$ & 0.35 \\
\hline
\end{tabular}

Data are presented as mean \pm SD or $\mathrm{n}(\%)$, unless otherwise stated. Crs: compliance of the respiratory system; Rrs: resistance of the respiratory system; FEV1: forced expiratory volume in 1s. " : higher vocational or university education.

\begin{tabular}{|c|c|c|c|c|c|}
\hline \multirow[t]{2}{*}{ Model } & \multirow[t]{2}{*}{ Subjects n } & \multicolumn{2}{|c|}{ Complete cases } & \multicolumn{2}{|c|}{ Imputed data } \\
\hline & & IRR (95\% CI) & p-value & IRR $(95 \% \mathrm{CI})$ & p-value \\
\hline 1 & 1762 & $1.018(1.004-1.032)$ & 0.01 & $1.010(0.994-1.026)$ & 0.22 \\
\hline 2 & 1675 & $1.023(1.007-1.038)$ & $<0.01$ & $1.008(0.992-1.025)$ & 0.31 \\
\hline 3 & 1673 & 1.028 (1.012-1.044) & $<0.01$ & 1.010 (0.994-1.027) & 0.22 \\
\hline 4 & 1420 & 1.038 (1.021-1.055) & $<0.01$ & 1.009 (0.993-1.025) & 0.27 \\
\hline 5 & 1420 & 1.015 (0.998-1.032) & 0.09 & $0.999(0.981-1.016)$ & 0.87 \\
\hline
\end{tabular}

\section{Discussion}

In our study we saw an association between a higher maternal BMI and increased risk of wheezing illnesses. This is the first study that explored whether neonatal lung function could be an intermediate factor in the association between maternal BMI and wheezing illnesses in the offspring. Our results showed that lung 
TABLE 3 Association between maternal body mass index and number of wheezing days in the offspring in the imputed data, stratified by maternal atopy

\begin{tabular}{|c|c|c|c|c|c|}
\hline \multirow[t]{2}{*}{ Model } & \multicolumn{2}{|c|}{ No maternal atopy } & \multicolumn{2}{|c|}{ Maternal atopy } & \multirow{2}{*}{$\begin{array}{c}p \text {-value } \\
\text { interaction }\end{array}$} \\
\hline & IRR (95\% CI) & p-value & IRR $(95 \% \mathrm{CI})$ & p-value & \\
\hline 1 & 1.027 (1.007-1.048) & 0.01 & 0.978 (0.951-1.005) & 0.11 & $<0.01$ \\
\hline 2 & $1.020(1.000-1.042)$ & 0.05 & $0.980(0.953-1.008)$ & 0.16 & $<0.01$ \\
\hline 3 & $1.023(1.003-1.045)$ & 0.03 & $0.981(0.953-1.010)$ & 0.19 & $<0.01$ \\
\hline 4 & $1.022(1.001-1.043)$ & 0.04 & 0.980 (0.953-1.008) & 0.16 & $<0.01$ \\
\hline 5 & 1.010 (0.988-1.033) & 0.38 & 0.976 (0.949-1.004) & 0.09 & 0.03 \\
\hline
\end{tabular}

IRR: incidence rate ratio; 1: univariable; 2: daycare, breastfeeding, older siblings, smoke exposure during pregnancy, pet keeping, socioeconomic status, ethnicity and maternal age; 3: all variables of model 2 and birthweight; 4: all variables of model 2 and compliance of the respiratory system; 5 : all variables of model 2 and resistance of the respiratory system.

function could partly explain the association between maternal BMI and increased risk of wheezing in the offspring in their first year of life, especially in children of nonatopic mothers. Even at the age of 5 years, infant lung function could explain part of the association with consultations for wheezing illnesses. However, weight and height of the child at the age of 5 years seemed to play a greater role. Maternal BMI was also associated with increased number of prescriptions for short-acting $\beta_{2}$-agonists and inhaled corticosteroids. It was remarkable that these associations, especially in children of atopic mothers, could not be explained by any of the potential confounders or intermediate factors. Whether disease status is better reflected by consultations or prescriptions for respiratory illnesses is unknown. Probably, children with well-controlled asthma have more prescriptions than consultations for wheezing illnesses. A hypothesis is that children with a lower baseline lung function have symptoms of wheezing illnesses, which are more difficult to control with medication and therefore have more GP consultations. Of all children with prescriptions of short-acting $\beta_{2}$-agonists and consultations for wheezing illnesses, the children with more consultations than prescriptions had a higher resistance in infancy (7.07 versus $7.93, \mathrm{p}<0.01)$; however this was not the case for prescriptions of inhaled corticosteroids.

Our findings of increased respiratory illness in the offspring of mothers with a higher maternal BMI are in line with previous literature $[5,6,9,20,21]$. Familial predisposition could be an effect modifier of the association; however the results of previous studies are inconsistent $[5,7,20]$. It is remarkable that we saw that in infants the associations were only present in the children of nonatopic mothers, while we observed a particularly increased risk of maternal BMI on wheezing illnesses in children at the age of 5 years if mothers had a history of atopy. This is in line with other studies in childhood [5, 20]. In infancy, a higher resistance is an important contributing factor to the development of wheeze. However, at an older age other pathophysiological mechanisms could contribute to the development of wheezing illnesses, which could explain the differences found. Children of atopic mothers could have a genetic predisposition with an increased sensitivity to inflammatory markers. If maternal states of heightened inflammation from higher fat mass transfer to the offspring, these particular children with a familial burden of atopy could be more susceptible to developing wheezing illnesses.

TABLE 4 Association between maternal body mass index and number of consultations and prescriptions for wheezing illnesses up to the age of 5 years in the offspring

\begin{tabular}{|c|c|c|c|c|c|c|c|c|}
\hline \multirow[t]{3}{*}{ Model } & \multirow[t]{3}{*}{ Subjects $n$} & \multirow{2}{*}{\multicolumn{2}{|c|}{$\begin{array}{l}\text { Consultations for } \\
\text { wheezing illnesses }\end{array}$}} & \multirow[t]{3}{*}{ Subjects $\mathbf{n}$} & \multicolumn{4}{|c|}{ Prescriptions of: } \\
\hline & & & & & \multicolumn{2}{|c|}{ Inhaled corticosteroids } & \multicolumn{2}{|c|}{ Short-acting $\boldsymbol{\beta}_{2}$-agonists } \\
\hline & & IRR $(95 \% \mathrm{CI})$ & p-value & & IRR $(95 \%$ CI) & $p$-value & $\operatorname{IRR}(95 \% \mathrm{CI})$ & $\mathrm{p}$-value \\
\hline 1 & 1033 & 1.035 (1.009-1.062) & 0.01 & 1051 & $1.076(1.047-1.106)$ & $<0.01$ & $1.046(1.023-1.069)$ & $<0.01$ \\
\hline 2 & 873 & 1.033 (1.001-1.065) & 0.04 & 888 & 1.097 (1.063-1.133) & $<0.01$ & $1.057(1.031-1.084)$ & $<0.01$ \\
\hline 3 & 524 & $1.002(0.962-1.044)$ & 0.92 & 524 & $1.109(1.061-1.160)$ & $<0.01$ & $1.073(1.036-1.111)$ & $<0.01$ \\
\hline
\end{tabular}

IRR: incidence rate ratio; 1: univariable; 2: daycare, breastfeeding, older siblings, smoke exposure during pregnancy, pet keeping, socioeconomic status, ethnicity and maternal age; 3 : all variables of model 2 and weight and height at the 5-year visit; 4: all variables of model 2 and resistance of the respiratory system. 
TABLE 5 Association between maternal body mass index and number of consultations and prescriptions for wheezing illnesses until the age of 5 years in the offspring, stratified by maternal atopy

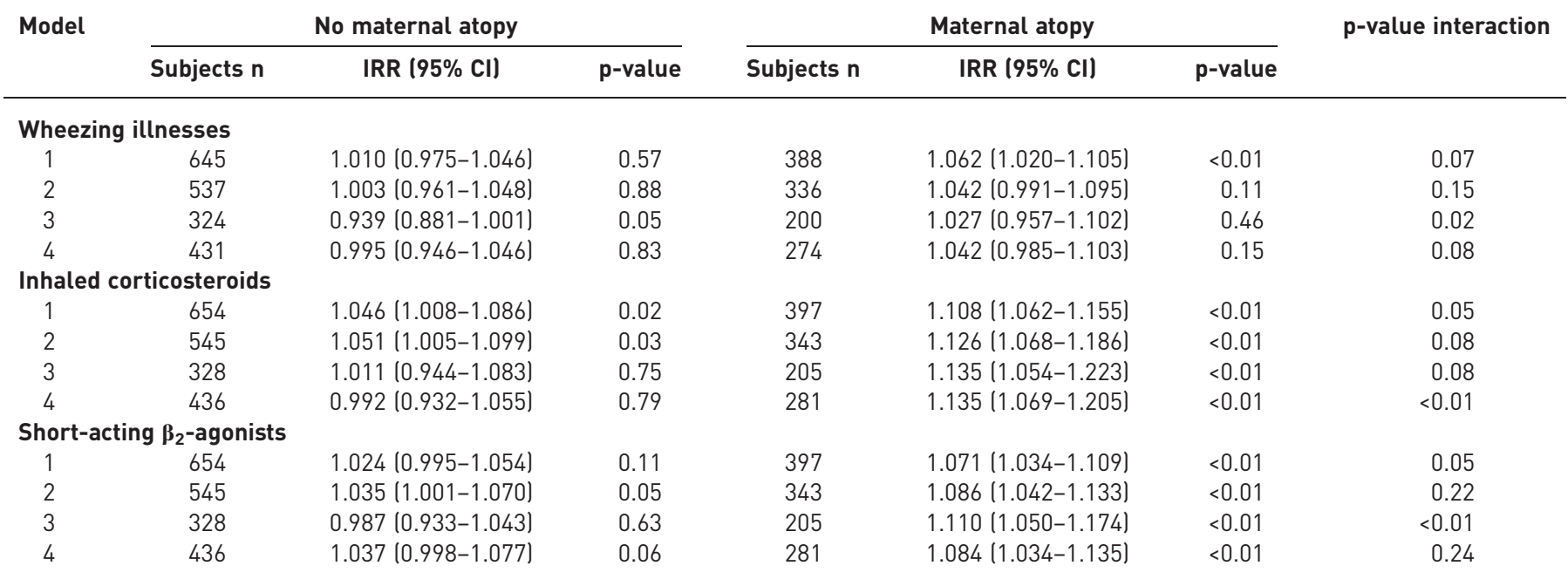

IRR: incidence rate ratio; 1: univariable; 2: daycare, breastfeeding, older siblings, smoke exposure during pregnancy, pet keeping, socioeconomic status, ethnicity and maternal age; 3: all variables of model 2 and weight and height at 5-year visit; 4: all variables of model 2 and resistance of the respiratory system.

Our study provides further evidence that potential confounders could not explain the associations. However, unknown confounders could still play a role. An additional analysis with adjustment for birth length and gestational age showed that these factors did not affect the associations in infancy (online supplementary table S2). Previous research has shown that intermediate factors, like pregnancy complications and postnatal growth, do not affect the association either $[5,9]$. In our study we adjusted for postnatal growth and current lung function at the age of 5 years, which confirmed these results (online supplementary table S3).

The current study has several strengths. Lung function data, measured before the age of 2 months, was available in >2000 infants. The sample size of our study is large and data were collected in a standardised manner. Data on wheezing in the first year of life were collected on a daily basis and prescriptions and GP consultations were derived from GP electronic patient files. Most previous studies were based on parent-reported symptoms derived from a retrospective questionnaire, which increases the risk of recall bias and parental misclassification $[5,6,9,20,21]$. We obtained information on wheezing in the first year of life from daily questionnaires with parent-reported symptoms. We minimised the risk of misclassification by careful parental instruction. Due to the prospective data collection, recall bias in our study is of no (or minor) influence. Besides parent-reported symptoms, we were also able to study the association with data on consultations and prescriptions for respiratory illnesses derived from the GP electronic patient files up to offspring age of 5 years.

Some limitations of the current study need consideration. First, bias due to selective follow-up could affect the association. Especially in the first year of life, where a lot of effort on behalf of the parents was requested, selected children could have missing data. We imputed the data of the children with no complete follow-up in their first year of life. At the age of 5 years, we assume selection bias will not have had a large impact on our association. Each child participating in WHISTLER and reaching the age of 5 years was re-invited for a follow-up visit. Selection bias due to selective follow-up is of concern if the association studied differs between those included and those not included in the analysis. Although we cannot study this association, it seems unlikely, as many children at the age of 5 years are lost to follow-up because they have moved and we did not observe a large difference in mean maternal BMI between children included at baseline and at the age of 5 years (maternal BMI 24.7 and $24.8 \mathrm{~kg} \cdot \mathrm{m}^{-2}$, respectively). Other baseline characteristics did not show large differences either (online supplementary table S1).

Second, weight and height of the parents were self-reported, which could have introduced an underestimation of those with a higher BMI. However, almost a third of the parents participated in the UHP and weight and height were measured in these parents. A sensitivity analysis including only mothers with BMI calculated from actual measured weight and height resulted in similar results (online supplementary table S4).

As our study has shown that infant lung function could partially explain the associations in early life, especially in children of nonatopic mothers, it suggests that there could be a direct negative effect of factors 
that are elevated in mothers with a higher maternal BMI on the structure of the respiratory system in the offspring. The exact mechanism needs to be elucidated, but as previous studies have shown that leptin plays a role in lung development, it would be interesting to further explore this. Of course, other adipokines, inflammatory or immunological factors could also play a role in this mechanism. Given the strong associations between maternal BMI and wheezing illnesses and the high prevalence of respiratory symptoms in childhood, it would be useful to gain more knowledge about the exact mechanism. A causal relationship could provide new targets for preventive interventions, as maternal obesity could be an important modifiable risk factor. Reducing weight in women of childbearing age could therefore improve health of both mother and child.

\section{Acknowledgements}

The authors gratefully acknowledge all the parents and children who participated in the study, Rolien Bekkema, Anne van der Gugten and Liesbeth van der Feltz-Minkema (Dept of Paediatric Pulmonology, Wilhemina Children's Hospital, University Medical Center, Utrecht, The Netherlands) for their major contribution to the data collection and Myriam Olling-de Kok for secretarial support.

\section{References}

1 Drake AJ, Reynolds RM. Impact of maternal obesity on offspring obesity and cardiometabolic disease risk. Reproduction 2010; 140: 387-398.

2 Reynolds RM, Allan KM, Raja EA, et al. Maternal obesity during pregnancy and premature mortality from cardiovascular event in adult offspring: follow-up of 1323275 person years. BMJ 2013; 347: f4539.

3 Ramsay JE, Ferrell WR, Crawford L, et al. Maternal obesity is associated with dysregulation of metabolic, vascular, and inflammatory pathways. J Clin Endocrinol Metab 2002; 87: 4231-4237.

4 Fraser A, Tilling K, Macdonald-Wallis C, et al. Association of maternal weight gain in pregnancy with offspring obesity and metabolic and vascular traits in childhood. Circulation 2010; 121: 2557-2564.

5 Leermakers ETM, Sonnenschein-van der Voort AMM, Gaillard R, et al. Maternal weight, gestational weight gain and preschool wheezing: the Generation R Study. Eur Respir J 2013; 42: 1234-1243.

6 Harpsøe MC, Basit S, Bager P, et al. Maternal obesity, gestational weight gain, and risk of asthma and atopic disease in offspring: a study within the Danish National Birth Cohort. J Allergy Clin Immunol 2013; 131: $1033-1040$.

7 Patel SP, Rodriguez A, Little MP, et al. Associations between pre-pregnancy obesity and asthma symptoms in adolescents. J Epidemiol Community Health 2012; 66: 809-814.

8 Pike KC, Inskip HM, Robinson SM, et al. The relationship between maternal adiposity and infant weight gain, and childhood wheeze and atopy. Thorax 2013; 68: 372-379.

9 Håberg SE, Stigum H, London SJ, et al. Maternal obesity in pregnancy and respiratory health in early childhood. Paediatr Perinat Epidemiol 2009; 23: 352-362.

10 Eising JB, Uiterwaal CSPM, Evelein AMV, et al. Relationship between leptin and lung function in young healthy children. Eur Respir J 2014; 43: 1189-1192.

11 Van der Gugten AC, Uiterwaal CSPM, van Putte-Katier N, et al. Reduced neonatal lung function and wheezing illnesses during the first 5 years of life. Eur Respir J 2013; 42: 107-115.

12 Katier N, Uiterwaal CSPM, de Jong BM, et al. The Wheezing Illnesses Study Leidsche Rijn (WHISTLER): rationale and design. Eur J Epidemiol 2004; 19: 895-903.

13 Grobbee DE, Hoes AW, Verheij TJM, et al. The Utrecht Health Project: optimization of routine healthcare data for research. Eur J Epidemiol 2005; 20: 285-287.

14 Katier N, Uiterwaal CSPM, de Jong BM, et al. Passive respiratory mechanics measured during natural sleep in healthy term neonates and infants up to 8 weeks of life. Pediatr Pulmonol 2006; 41: 1058-1064.

15 Katier N, Uiterwaal CSPM, de Jong BM, et al. Feasibility and variability of neonatal and infant lung function measurement using the single occlusion technique. Chest 2005; 128: 1822-1829.

16 Gappa M, Colin AA, Goetz I, et al. Passive respiratory mechanics: the occlusion techniques. Eur Respir J 2001; 17: 141-148.

17 Frey U, Stocks J, Coates A, et al. Specifications for equipment used for infant pulmonary function testing. Eur Respir J 2000; 16: 731-740.

18 Beydon N, Davis SD, Lombardi E, et al. An official American Thoracic Society/European Respiratory Society statement: pulmonary function testing in preschool children. Am J Respir Crit Care Med 2007; 175: 1304-1345.

19 Munnik P, Zanen P, Lammers JWJ. A comparison of lung function equipment with emphasis on interchangeability and methods. Physiol Meas 2006; 27: 445-455.

20 Scholtens S, Wijga AH, Brunekreef $\mathrm{B}$, et al. Maternal overweight before pregnancy and asthma in offspring followed for 8 years. Int J Obes 2010; 34: 606-613.

21 Guerra S, Sartini C, Mendez M, et al. Maternal prepregnancy obesity is an independent risk factor for frequent wheezing in infants by age 14 months. Paediatr Perinat Epidemiol 2013; 27: 100-108. 